\title{
EXPLORING MULTIPACTING CHARACTERISTICS OF A RECTANGULAR WAVEGUIDE*
}

\author{
R.L. Geng $^{\dagger \ddagger}$, H.S. Padamsee, Laboratory of Nuclear Studies, Cornell University, Ithaca, NY 14853 \\ Abstract

\section{THE CODE}

Multipacting (MP) characteristics of the rectangular waveguide used for CESR-III Superconducting RF (SRF) system were investigated by using a newly developed 3-D computer code. MP bands were identified for different operating modes, such as standing wave (SW) mode, traveling wave (TW) mode and the so called mixed wave mode (TW mixed with SW). A comparison between the simulation results and the experimental observations for the installed CESR-III SRF systems was made. Measures to suppress multipactor were explored. Our simulation results suggested that an external DC magnetic field of a few Gauss of amplitude in the wave propagation direction would be very effective in disturbing the trajectories. Further work will include disk-type windows in the waveguide couplers.

\section{INTRODUCTION}

Multipacting (MP) is a resonant electronic process that may happen when a system is subjected to RF fields and vacuum[1]. When MP occurs, a large number of electrons are generated, consuming the RF power in the system and resulting in vacuum degradation or discharge and metal sputtering.

The Multipacting phenomena for various systems have been studied intensively in the past few decades[2][3][4]. Scaling laws were established based on theoretical studies for simple geometries and have been successfully used for guiding designs. More recently, numerical simulations were performed to study MP in more complex RF structures for accelerators[5][6][7].

Waveguide input couplers are used for the PEP-II normal conducting RF system[8] and CESR-III SRF system[9]. These waveguides are expected to work in regimes which have not been encountered before, namely multi-hundred $\mathrm{kW}$ power levels and few hundred $\mathrm{MHz}$ frequencies. It is of interest to understand the MP characteristics and the influences of MP on the high power performance of these couplers. Basically two questions need to be answered: 1) are there any possibilities that MP may occur? 2) if there are possibilities what measures are available to stop the multipactor? To answer these questions, simple scaling laws are not enough and simulation codes are needed, because these couplers work not only in pure TW/SW modes, but also in a more complex mode, namely mixed wave mode. Also, it is highly desired to investigate MP in the ceramic window regions of these couplers. In this case no scaling law is handy and a numerical code is indispensable.

\footnotetext{
* Work supported by the National Science Foundation.

${ }^{\dagger}$ on leave from IHIP, Peking University, Beijing 100871, PRC.

‡Email: rg58@ cornell.edu
}

The simulation code essentially consists of two core parts, one for computing electron trajectories and the other for searching and analyzing MP. The leap-frog algorithm was used in the trajectory computing part and electron motions were described with relativistic dynamic equations. Note that the space-charge effect was not taken into account. For simplicity, it was assumed that the electron was emitted at an energy of $2 \mathrm{eV}$, with velocity perpendicular to the emission surface.

Denoting the wide and narrow dimensions of the reduced height waveguide in the CESR SRF system as $a(0.433 \mathrm{~m})$ and $b(0.102 \mathrm{~m})$, the electro-magnetic fields of the dominant mode $\left(T E_{10}\right)$ can be generally described in a $(x, y, z)$ coordinate system as follows,

$\left\{\begin{array}{l}E_{y}=E_{0} \sin (X)[\cos (\omega t-k z)+\rho \cos (\omega t+k z)], \\ B_{x}=\frac{-k}{\omega} E_{0} \sin (X)[\cos (\omega t-k z)-\rho \cos (\omega t+k z)], \\ B_{z}=\frac{-\pi}{\omega a} E_{0} \cos (X)[\sin (\omega t-k z)+\rho \sin (\omega t+k z)],\end{array}\right.$

where $E_{0}$ is the maximum electric field of the forward wave, $X=\pi x / a, \omega=2 \pi f(f=500 \mathrm{MHz})$ is the angular frequency of the field, $k=\sqrt{\omega^{2} \mu \epsilon-\left(\frac{\pi}{a}\right)^{2}}$ is the propagation constant, $t$ is time, and $\rho$ is the voltage reflection coefficient. $E_{0}$ is related to the forward RF power $\left(P_{f}\right)$ like this, $P_{f}=\frac{E_{0}^{2} a b k}{4 \omega \mu}$. $\rho$ can be derived from the forward and reflected $\left(P_{r}\right)$ powers, $\rho=\sqrt{\frac{P_{r}}{P_{f}}}$. Note that the fields are pure TW if $\rho=0$, pure SW if $\rho= \pm 1$, and mixed wave otherwise.

It is well known that MP characteristics depend strongly on the surface characteristics. In our simulation the secondary emission coefficient (SEC) of a "wet prepared" niobium[5] was used. For improving the computation efficiency the $\operatorname{SEC}(\delta)$ is formulated[10] as follows,

$$
\delta(u)=\delta_{m} \frac{1-\exp \left[-A\left(u / u_{m}\right)^{B}\right]}{C\left(u / u_{m}\right)^{D}},
$$

here $u$ in $\mathrm{eV}$ is the impacting energy of the primary electron and $\delta_{m}=1.6$ is the maximum SEC corresponding to an impacting energy of $u_{m}=200 \mathrm{eV}$. The curved fitted $A B C D$ parameters are $A=1.55, B=0.9, C=0.79$, and $D=$ 0.35 .

The very useful concepts of counter function and enhanced counter function were adopted in the MP searching and analyzing part. A complete description of these concepts can be found in Ref.[7]. Basically, the counter function is a measure of the number of survived primary electrons after several (e.g. 10 or 20) impacts, and the enhanced counter function is a measure of total number of electrons, including primaries and secondaries, after the same number 
of impacts. In the following sections, we will show relative enhanced counter function, $e_{n}$ where $\mathrm{n}$ is the number of impacts, as a function of the forward power. An $e_{n}$ which is larger than unity is a good indication of the existance of an MP band.

\section{MP BANDS FOR DIFFERENT WORKING MODES}

Due to symmetry, it is enough to consider a waveguide section with a length of a quarter guided wavelength. After a survey launching over the whole inner surface of such a section, it was found that only electrons launched from the peak electric site may develop into MP. So in the following discussions, electrons are launched from only the peak electric site(s).

The relative enhanced counter functions for 10 and 20 impacts for the SW mode and TW mode are shown in Fig. 1 - Fig. 4 respectively. Among all the MP bands, only a

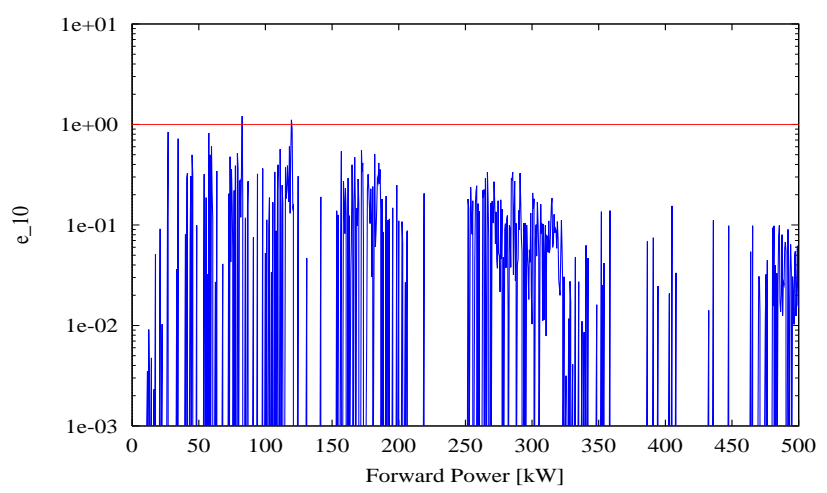

Figure 1: The relative enhanced counter function $e_{10}$ for the SW mode. The $e_{10}=1$ line is indicated.

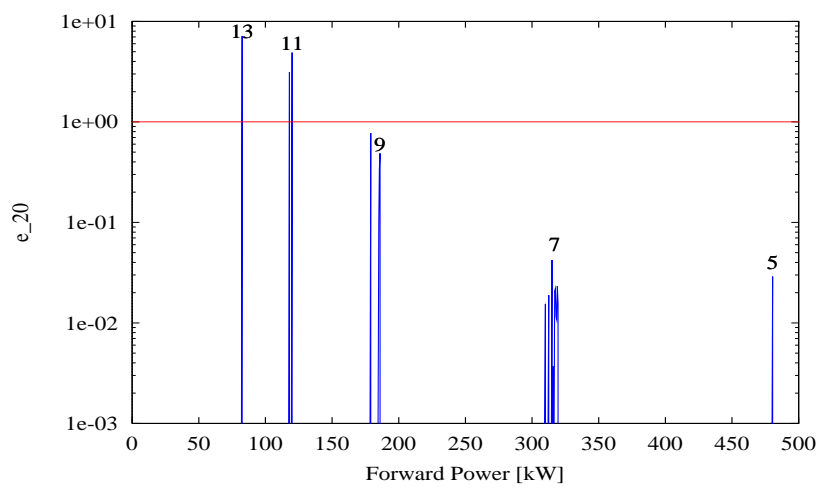

Figure 2: The relative enhanced counter function $e_{20}$ for the SW mode. The $e_{20}=1$ line is indicated.

few are robust enough to sustain 20, even more, impacts. All others are mild and can sustain only 10 impacts. The mild bands may appear when the surface's SEC is high but are expected to be easily processed. Robust bands could

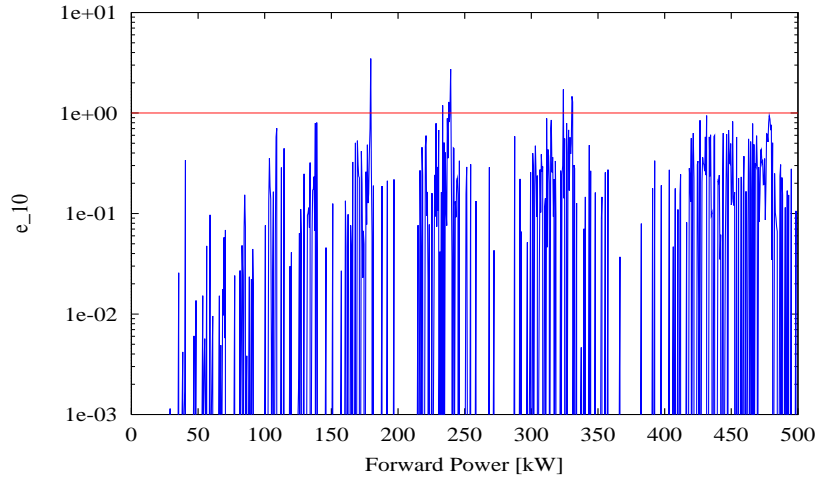

Figure 3: The relative enhanced counter function $e_{10}$ for the TW mode. The $e_{10}=1$ line is indicated.

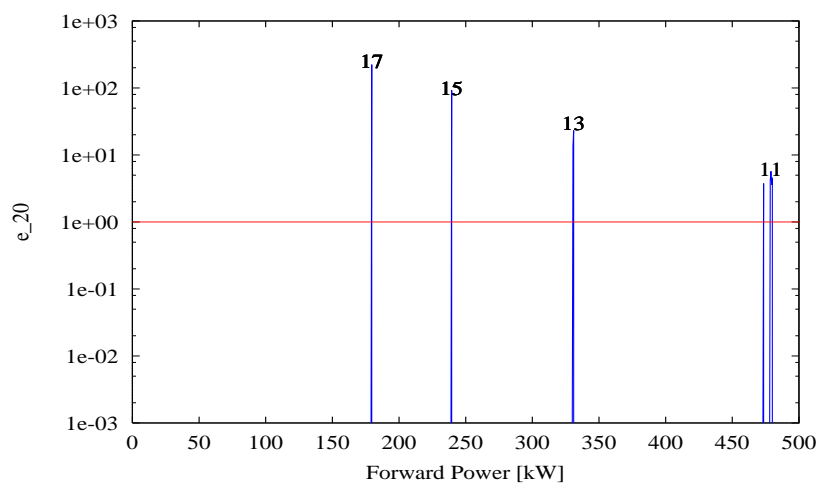

Figure 4: The relative enhanced counter function $e_{20}$ for the TW mode. The $e_{20}=1$ line is indicated.

constitute hard barriers. Further trajectory computations revealed that all those MP's are two-point MP. Electrons travel back and forth and impact with the bottom and top walls. For the SW mode, impacts are limited to two points on the bottom and top walls; while for the TW case, impacting points drift along the wave propagation direction. The MP order, number of half RF cycles elapsed between succeeding impacts, is also labeled in the plot of $e_{20}$.

Fig. 5 and Fig. 6 show the relative enhanced counter functions for 10 and 20 impacts for mixed waves with $1 \%$ and $4 \%$ reflected power mixed. Two main features manifest themselves as a result of trajectory disturbance due to wave mixing: 1) compared to the pure TW case (Fig. 3 and Fig. 4), the MP band at $90 \mathrm{~kW}$ is enhanced; 2) with more reflected power mixed, most MP bands are shifted up to higher power levels with lower intensities. These effects were experimentally confirmed during the machine studies of the first SRF cavity for CESR luminosity upgrade. A barrier was encountered at about $90 \mathrm{~kW}$ forward power. When the cavity was run at a $6.3 \mathrm{MV} / \mathrm{m}$ gradient, the forward power limit due to RF trip was $125 \mathrm{~kW}$; while at 7 $\mathrm{MV} / \mathrm{m}$, the forward power limit was pushed to $135 \mathrm{~kW}$. An extra $10 \mathrm{~kW}$ forward power was gained as a result of more reflected power mixed. 


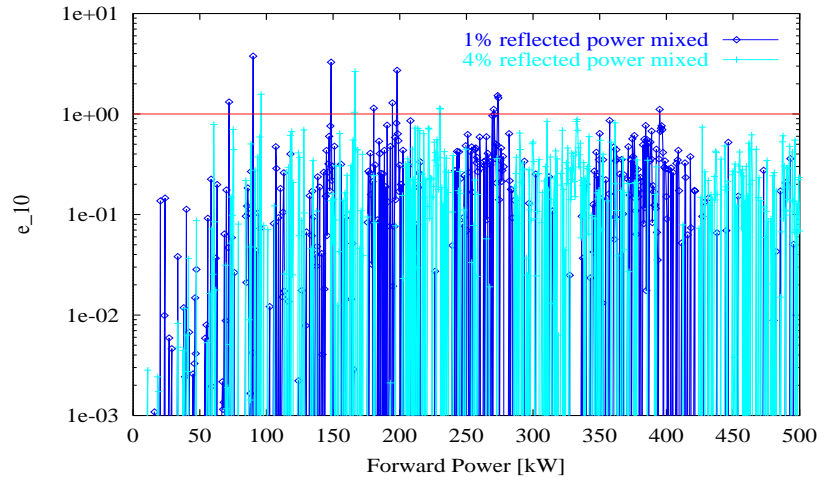

Figure 5: The relative enhanced counter function $e_{10}$ for mixed wave modes. The $e_{10}=1$ line is indicated.

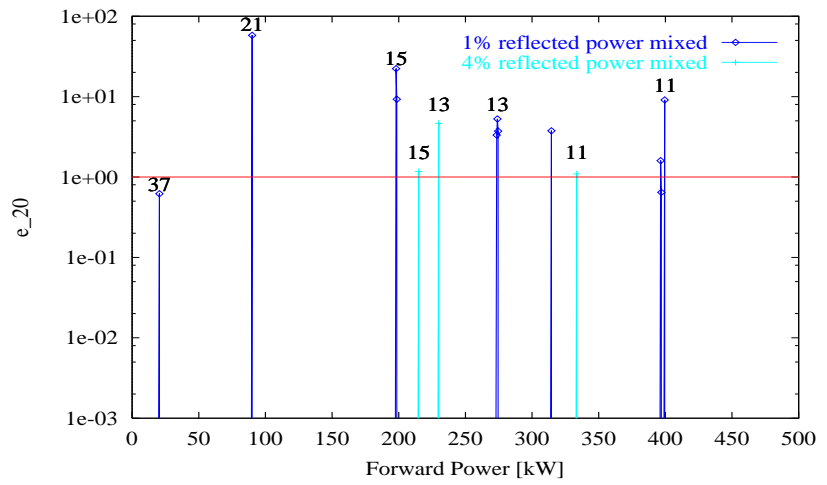

Figure 6: The relative enhanced counter function $e_{20}$ for mixed wave modes. The $e_{20}=1$ line is indicated.

\section{SUPPRESSING MP WITH PERTURBATION MAGNETIC FIELDS}

It is well known that MP can be avoided by applying suitable perturbation DC electric or magnetic fields. In case of a rectangular waveguide, a magnetic perturbation seems more natural for this purpose. Fig.7 shows the relative enhanced counter functions for 20 impacts with 3 Gauss magnetic perturbation fields applied along $x$ and $z$ direction for the TW mode. It is shown that with a 3 Gauss perturbation field along $x$ direction, the MP is enhanced, both in intensity and in bandwidth; while with a 3 Gauss field along $z$, all the MP bands bellow $500 \mathrm{~kW}$ are virtually eliminated. For the SW case, it is also justified that a perturbation magnetic field along $z$ direction is effective in suppressing MP.

\section{CONCLUSIONS AND OUTLOOK}

Below $500 \mathrm{~kW}$ forward power, there are a couple of clearly defined MP bands in the reduced height waveguide in the CESR-III SRF system. For the TW mode these bands are quite likely dangerous. For the SW mode, only the bands below $150 \mathrm{~kW}$ forward power seem harmful. Mixing a forward wave with some portion of a reflected wave will push the multipactor to higher power levels and MP intensities

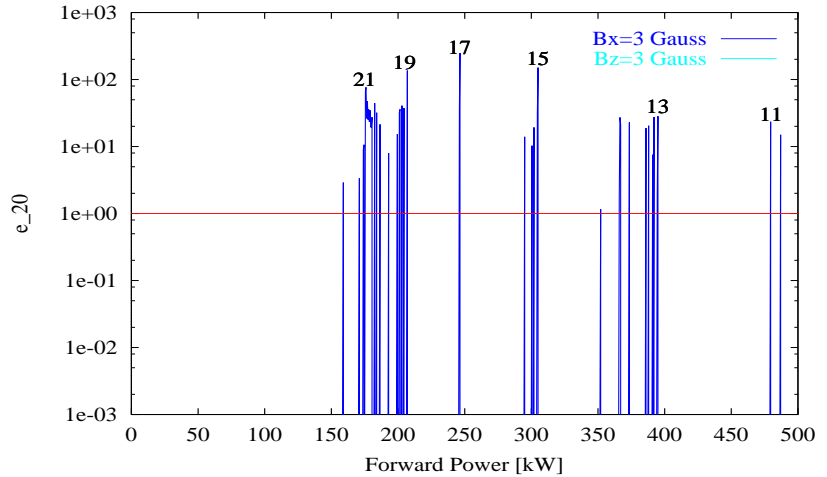

Figure 7: The relative enhanced counter functions $e_{20}$ for the TW mode with perturbation magnetic fields (note that the data for $B_{z}=3$ Gauss are too small, $\sim 10^{-10}$, to be shown). The $e_{20}=1$ line is indicated.

will decrease. A perturbation magnetic field along $x$ direction makes the MP enhanced; while a perturbation magnetic field along $z$ direction eliminates virtually all the MP bands bellow $500 \mathrm{~kW}$ forward power.

Currently we are studying MP in the ceramic window region in the waveguide. Due to the structure complexity in that region, the field distributions needed by the MP computation can only be obtained with a numerical code. A practical problem arises when computing MP with numerical fields due to space discretization. In order to work out a way to incorporate discretized fields into MP computations, the waveguide investigated above was studied again with MAFIA[11] field distributions. The main MP features were well repeated with a $3 \mathrm{~mm}$ mesh size, which gives us confidence to go on to complex structures like ceramic regions.

Finally, we would like to mention that the algorithm used in this paper was checked against analytical results for a parallel-plate and proved to be reliable.

\section{REFERENCES}

[1] H. Padamsee, J. Knobloch, and T. Hays, RF superconductivity for accelerators, John Wiley \& Sons, Inc. 1998.

[2] E.W.B. Gill and A. von Engel, Proc. R. Soc. London A192, 446(1948).

[3] A.J. Hatch and H.B. Williams, Phys. Rev. 112(3), 681(1958).

[4] A. Woode and J. Petit, ESA Journal 14(4), 467(1990).

[5] H. Padamsee and A. Joshi, J. Appl. Phys. 50(2), 1112(1979).

[6] S. Yamaguchi et. al., IEEE Trans. on Nucl. Sci. 39(2), 278(1992).

[7] E. Somersalo et. al., Particle Accelerators 59, 107(1998).

[8] M. Neubauer et. al., Proc. 1995 Part. Accel. Conf. p.1803.

[9] E. Chojnacki et. al., Proc. of the $8^{\text {th }}$ Workshop on RF Superconductivity, Abano Terme, Italy, 1997.

[10] G.E. Dionne, J. Appl. Phys. 46, 3347(1975).

[11] http://www.cst.de/ 